Pacific Journal of Mathematics

THE TAUBERIAN THEOREM FOR GROUP ALGEBRAS O 


\title{
THE TAUBERIAN THEOREM FOR GROUP ALGEBRAS OF VECTOR-VALUED FUNCTIONS
}

\author{
Alvin Hausner
}

1. Introduction. The object of this paper is to prove the idealtheoretic version of Wiener's tauberian theorem for algebras which we will call group algebras of vector-valued functions. These algebras are defined as follows. Let $G=\{a, b, \cdots\}$ denote a locally compact abelian group and let $X=\{x, y, \cdots\}$ represent a complex commutative Banach algebra. Our group algebra $B=B(G, X)$ consists of the set of all measurable absolutely integrable functions defined over $G$ with values in $X$. Of course we must identify functions which differ on sets of Haar measure 0. As norm for an element $f \in B$ we take

$$
\|f\|_{B}=\int_{G}|f(a)|_{X} d a .
$$

(Hereafter, we will omit an indication of the domain of integration if the integral is taken over the entire group $G$.) The space $B(G, X)$ is known to be complete in the given norm [4]. Further, we introduce into $B$ the following operations

$$
(f+g)(a)=f(a)+g(a), \quad(\lambda f)(a)=\lambda f(a)
$$

where $\lambda$ is a complex number, and

$$
(f * g)(a)=\int f(b) g(a-b) d b
$$

where the integral is taken in the sense of Bochner $[1,4]$ with respect to Haar measure $d b$. The algebra $B(G, X)$ thus becomes, as is easily shown, a complex commutative Banach algebra which specializes into the classical group algebra $L(G)$ if $X$ is chosen as the complex numbers. It is these algebras $B(G, X)$ which will be the object of our study.

The tauberian theorem for $B(G, X)$ will be proved by appealing to a theorem in the general theory of Banach algebras (see [5], p. 85 corollary, or [6], Theorem 38.) This latter result might be designated as the "general tauberian theorem." It says that if a complex commutative $B$-algebra $Y$ is semi-simple, regular, and is such that the set of $y \in Y$ with $\phi_{M}(y)$ having compact support in $\mathfrak{M}(Y)$ is dense in $Y$, then every proper closed ideal in $Y$ is contained in a regular maximal ideal.

Received November 7, 1955 and in revised form February 5, 1957. This paper is a revised version of a portion of the author's Yale (1955) doctoral dissertation. See, also, $[\mathbf{3}]$ in the references at the end of the paper. 
Here $\mathfrak{M}(Y)$ denotes the space (in the usual weak topology) of regular maximal ideals in $Y$ and $\phi_{M}$ represents the canonical homomorphism from $Y$ onto the complex numbers associated with an $M \in \mathfrak{M}(Y)$. It will be taken as known that the classical group algebra $L(G)$ satisfies the hypotheses of this general tauberian theorem. This amounts, then, to assuming the tauberian theorem in the case of $L(G)$. It will also be assumed, but only in the final theorem of the paper, that the range space $X$ meets the conditions of the general tauberian theorem. It is clear, therefore, that the proof of the tauberian theorem for $B(G, X)$ found here, does not yield a new proof in the case of $L(G)$. However, this paper does provide, it is hoped, an interesting application of the general tauberian theorem in the case of our generalized algebras.

2. Proof of the theorem. It is important to know the form of the most general multiplicative linear functional in $B(G, X)$. This is determined in Lemma 1 which requires the following preliminary observations.

The convolution $f * g$ of a function $f \in L(G)$ with a function $g \in B(G, X)$ results, as in easily seen, in a function contained in $B(G, X)$. Suppose $\left\{j_{W}\right\}$ is an approximate identity for $L(G)$; that is, for each neighborhood $W$ of the identity 0 in $G, j_{W}$ is some (numerical) non-negative function vanishing off $W$ such that $\int j_{W}(a) d a=1$. Then for every $f \in L(G)$ we have $j_{W} * f \rightarrow f$ as $W \rightarrow 0$. (Of course, convergence is here understood in the sense of directed systems.) But $\left\{j_{W}\right\}$ acts, also, as an approximate identity in $B(G, X)$, that is, $j_{W} * g \rightarrow g$ in $B$-norm for every $g \in B$. This can be shown, just as in the case of $L(G)$, by noting that functions in $B$ are continuous in $B$-norm [4], i.e., for any $\varepsilon>0$ there is a neighborhood $W_{\varepsilon}$ of 0 in $G$ such that $\|f(a-b)-f(a)\|_{B}<\varepsilon$ if $b \in W_{\varepsilon}$.

The approximate identity will be of service to us in proving Lemma 1 which we now state.

Lemma 1. Let $\hat{G}=\{\hat{a}, \hat{b}, \cdots\}$ denote the dual group of $G$ in the usual Pontrjagin topology. Define the "Fourier transform" of $f \in B$ as

$$
\hat{f}(M, \hat{a})=\int \phi_{M} f(a)(a, \hat{a}) d a .
$$

The Fourier transform evaluated at a fixed $(M, \hat{a}) \in \mathfrak{M}(X) \times \hat{G}$ is a nonzero, continuous multiplicative linear functional in $B$ and, further, all such functionals are of this type, that is, if $\mu$ is a non-zero, continuous multiplicative linear functional in $B$, then there is some $(M, \hat{a})$ such that $\mu(f)=\hat{f}(M, \hat{a})$ for every $f \in B$. 
Proof. That the Fourier transform, at a fixed $(M, \hat{a})$, is a multiplicative functional is easily shown. We, consequently, turn to the second half of the lemma. Choose a function $f \in B$ such that $\mu(f) \neq 0$ and let $\left\{j_{W}\right\}$ be an approximate identity. For every $x \in X, \lim _{W \rightarrow 0} \mu\left(j_{W} x\right)$ exists. (Here, $j_{W} x$ denotes the function $\left(j_{W} x\right)(a)=j_{W}(a) \cdot x$. Of course, $j_{W} x \in B$.) For

$$
\mu\left(j_{W} x * f\right)=\mu\left(j_{W} x\right) \mu(f)=\mu\left[\left(j_{W} * f\right) x\right] \rightarrow \mu(f x) \quad \text { as } \quad W \rightarrow 0
$$

because $\left(j_{W} * f\right) x \rightarrow f x$. Hence $\mu\left(j_{W} x\right)$ necessarily converges to a limit independent of the approximate identity $\left\{j_{W}\right\}$, namely $\mu(f x) / \mu(f)$. This limit is likewise independent of the $f \in B$ with $\mu(f) \neq 0$, for if $g \in B$ is such that $\mu(g) \neq 0$, then

$$
\mu(f x) \mu(g)=\mu[(f * g) x]=\mu(g x * f)=\mu(g x) \mu(f)
$$

so that $\mu(f x) / \mu(f)=\mu(g x) / \mu(g)$. We will denote the limit of $\mu\left(j_{W} x\right)$ by $\phi_{\mu}(x)$ for $x \in X$.

Suppose, temporarily, that $X$ possesses an identity $e$. Then $\phi_{\mu}$ is certainly not zero. For $\phi_{\mu}(e)=\mu(f e) / \mu(f)=\mu(f) / \mu(f)=1$. Further, $\phi_{\mu}$ is easily seen to be additive and homogeneous, that is,

$$
\phi_{\mu}\left(\lambda_{1} x+\lambda_{2} y\right)=\lambda_{1} \phi_{\mu}(x)+\lambda_{2} \phi_{\mu}(y)
$$

for all $x, y \in X$ and complex numbers $\lambda_{1}, \lambda_{2}$.

$$
\phi_{\mu}(x y)=\frac{\mu(f * f \cdot x y)}{\mu(f * f)}=\frac{\mu(f x)}{\mu(f)} \cdot \frac{\mu(f y)}{\mu(f)}=\phi_{\mu}(x) \phi_{\mu}(y),
$$

so that $\phi_{\mu}$ is multiplicative. Therefore, as is well known, there is some $M \in \mathfrak{M}(X)$ (depending on $\mu$ ) such that $\phi_{\mu}(x)=\phi_{M}(x)$.

Still assuming that $X$ has an $e$ (which we may take of norm 1), let $g \in L(G), x \in X$. Then

$$
\mu\left(j_{W} * g x\right)=\mu\left(j_{W} x * g e\right)=\mu\left(j_{W} x\right) \mu(g e) \rightarrow \phi_{M}(x) \mu(g e) .
$$

But

$$
\mu\left(j_{W} * g x\right)=\mu\left[\left(j_{W} * g\right) x\right] \rightarrow \mu(g x)
$$

so that $\mu(g x)=\phi_{M}(x) \mu(g e)$ for any $g \in L(G)$ and any $x \in X$. Since $L e=\{g e \in B \mid g \in L(G)\}$ is isometrically isomorphic with $L(G)$ and since $\mu$ is a continuous multiplicative linear functional on $L e \subset B$ (not identically zero on $L e$, because linear combinations of functions $g x$ with $g \in L(G)$, $x \in X$ are dense in $B[1,4]$ ) there is an $\hat{a} \in \hat{G}$ (depending on $\mu$ ) such that $\mu(g e)=\int g(a)(a, \hat{a}) d a$ for all $g \in L(G)$.

Suppose, now, that $f$ is any function in $B$. Then, because the 
simple functions are dense in $B(G, X)$ as we observed above, there exists a sequence $g_{n} \in B$ such that $g_{n} \rightarrow f$ and $\mu\left(g_{n}\right)=\hat{g}_{n}(M, \hat{a}) \rightarrow \hat{f}(M, \hat{a})$ and so $\mu(f)=\hat{f}(M, \hat{a})$.

We now remove the restriction that $X$ possess an identity. If $X$ lacks an $e$, then we imbed $X$, isometrically and isomorphically, in a Banach algebra $X^{\prime}$ with unit $e$ in such a way that maximal ideals in $X^{\prime}$ are the regular maximal ideals in $X$ and $X$ itself. This is done in the usual well-known manner. The homomorphisms of $X^{\prime}$ onto the complex numbers are $\phi_{M}(M \in \mathfrak{M}(X))$ and the additional functional $\phi_{X}$, where $\phi_{X}(x+\lambda e)=\lambda$ for $x \in X, \lambda$ a complex number. By what we have already proved, the non-zero multiplicative functionals in $B\left(G, X^{\prime}\right)$ are of the form $\hat{f}(M, \hat{a})$ and the additional functionals $\hat{f}(X, \hat{a})$. These latter functionals, namely, $\int \phi_{X} f(a)(a, \hat{a}) d a$ are, however, all identically zero in $B(G, X)$ and thus the lemma is established.

The following lemma gives a topological characterization of the space of regular maximal ideals $\operatorname{Ml}_{(}(B)$ in $B(G, X)$. For a similar result and proof see [2].

Lemma 2. The space $\mathfrak{M}(B)$ of regular maximal ideals in $B$, topologized in the weak topology, is homeomorphic with $\mathfrak{M}(X) \times \hat{G}$, that is the topological product of $\mathfrak{M}(X)$ and $\hat{G}$.

Proof. There is a $1-1$ correspondence between the points of $\mathfrak{M}(B)$ and those of $\mathfrak{M}(X) \times \hat{G}$. To see this, suppose $(M, \hat{a}) \neq(N, \hat{b})$. If $\hat{a} \neq \hat{b}$ and $M=N$, take $x \notin M$ and find an $f \in L(G)$ such that

$$
\hat{f}(\hat{a})=\int f(a)(a, \hat{a}) d a \neq \hat{f}(\hat{b}) \text {. }
$$

Then

$$
\hat{f x}(M, \hat{a})=\hat{f}(\hat{a}) \phi_{M}(x) \neq \hat{f x}(N, \hat{b}) .
$$

If $\hat{a} \neq \hat{b}$ and $M \neq N$ or if $\hat{a}=\hat{b}$ and $M \neq N$, then we may proceed in the same way to construct a function $f x$ with $f \in L(G), x \in X$ such that the Fourier transform of $f x$ separates the points $(M, \hat{a}),(N, \hat{b})$. No two points in $\mathfrak{M}(X) \times \hat{G}$ give rise to the same regular maximal ideal in $\mathfrak{M}(B)$.

The topology of $\mathfrak{M}(B)$ is precisely that induced by the family $\mathfrak{s}=\{\hat{f}(M, \hat{a}) \mid f \in B\}$ of functions defined on $\mathfrak{M}(X) \times \hat{G}$. We must show that this topology is identical with the product topology of $\mathfrak{M}(X) \times \hat{G}$. This will be done by showing that the $\Im$-topology of $\mathfrak{M}(X) \times \hat{G}$ is iden- 
tical with that induced by another family of functions $\mathfrak{F} \subset \mathfrak{F}$ defined on $\mathfrak{M}(X) \times \hat{G}$. Then the proof will be completed by showing that this $\mathfrak{F}$ topology is identical with the product topology of $\mathfrak{M}(X) \times \hat{G}$.

First we must define $\widetilde{F}$. For each positive integer $n$ and each choice $f_{1}, f_{2}, \cdots, f_{n} \in L(G) ; x_{1}, x_{2}, \cdots, x_{n} \in X$, there is a function $\hat{h}$ defined on $\mathfrak{M}_{(}(X) \times \hat{G}$ by $\hat{h}(M, \hat{a})=\sum_{i=1}^{n} \widehat{f_{i} x_{i}}(M, \hat{a})$, Let $\mathfrak{F}$ be the family of all functions $\hat{h}$ so defined. Clearly $\mathfrak{F} \subset \mathfrak{F}$. But $\mathfrak{F}$ is also dense in $\mathfrak{\Im}$ in the uniform norm. For, suppose $f \in B$. Then we can find $f_{i} \in L(G)$, $x_{i} \in X$, such that $\left\|f-\sum_{i=1}^{n} f_{i} x_{i}\right\|_{B}<\varepsilon$. Hence

$$
\left|\hat{f}(M, \hat{a})-\sum_{i=1}^{n} \widehat{f_{i} x_{i}}(M, \hat{a})\right|=\left|\int\left[\phi_{M} f(a)-\sum_{i=1}^{n} f_{i}(a) \phi_{M}\left(x_{i}\right)\right](a, \hat{a}) d a\right|<\varepsilon .
$$

Therefore, $\sup \left|\hat{f}(M, \hat{a})-\sum_{i=1}^{n} \widehat{f_{i} x_{i}}(M, \hat{a})\right| \leqq \varepsilon$ where the sup is taken over $\mathfrak{M}(X) \times \hat{G}$. This shows $\mathfrak{F}$ is dense in $\mathfrak{\Im}$ in the sup-norm and it is easy to see, from this, that the $\mathfrak{F}$ - and $\Im$-topologies on $\mathfrak{M}(X) \times \hat{G}$ are identical.

It remains to show that the $\mathfrak{F}$-topology on $\mathfrak{M}(X) \times \hat{G}$ is the same as the product topology. To do this we first develop a few properties of $\mathfrak{F}$.

(i) The functions in $\widetilde{F}$ separate the points of $\mathfrak{M}(X) \times \hat{G}$ as we saw in the beginning of this proof.

(ii) Functions in $\mathfrak{F}$ are continuous over $\mathfrak{M}(X) \times \hat{G}$ in the product topology. For, if $f \in L(G), x \in X,\left(M_{0}, \hat{a}_{0}\right)$ is a fixed point of $\mathfrak{M}(X) \times \hat{G}$, and $\varepsilon>0$, then

$$
\begin{aligned}
\mid \hat{f x}(M, \hat{a}) & -\hat{f x}\left(M_{0}, \hat{a}_{0}\right) \mid \\
& \leqq\left|\hat{f}(\hat{a}) \phi_{M}(x)-\hat{f}(\hat{a}) \phi_{M_{0}}(x)\right|+\left|\hat{f}(\hat{a}) \phi_{M_{0}}(x)-\hat{f}\left(\hat{a}_{0}\right) \phi_{M_{0}}(x)\right| \\
& =|\hat{f}(\hat{a})| \cdot\left|\phi_{M}(x)-\phi_{M_{0}}(x)\right|+\left|\phi_{M_{0}}(x)\right| \cdot\left|\hat{f}(\hat{a})-\hat{f}\left(\hat{a}_{0}\right)\right| \\
& \leqq|f|_{L} \cdot\left|\phi_{M}(x)-\phi_{M_{0}}(x)\right|+|x| \cdot\left|\hat{f}(\hat{a})-\hat{f}\left(\hat{a}_{0}\right)\right| \\
& <|f|_{L} \cdot\left(\varepsilon / 2|f|_{L}\right)+|x| \cdot(\varepsilon / 2|x|)=\varepsilon
\end{aligned}
$$

if $(M, \hat{a}) \in U\left(M_{0}\right) \times \hat{U}\left(\hat{a}_{0}\right)$ where $\hat{U}\left(M_{0}\right), \quad \hat{U}\left(\hat{a}_{0}\right)$ are neighborhoods of $M_{0}$, $\hat{a}_{0}$ in $\mathfrak{M}(X)$ and $\hat{G}$, respectively, such that $\left|\phi_{M}(x)-\phi_{M_{0}}(x)\right|<\varepsilon / 2|f|_{L}$ for $M \in U\left(M_{0}\right)$ and $\left|\hat{f}(\hat{a})-\hat{f}\left(\hat{a}_{0}\right)\right|<\varepsilon / 2|x|$ for $\hat{a} \in \hat{U}\left(\hat{a}_{0}\right)$. Since $\widetilde{\mho}$ consists of finite linear combinations of $\hat{f x}(f \in L(G), x \in X)$, each function in $\widetilde{F}$ is continuous in the product topology of $\mathfrak{M}(X) \times \hat{G}$.

(iii) Let $\left(M_{0}, \hat{a}_{0}\right) \in \mathfrak{M}(X) \times \hat{G}$. Choose $f \in L(G)$ such that $\hat{f}\left(\hat{a}_{0}\right) \neq 0$ 
and let $x \in X$ be such that $x \notin M_{0}$. Then $\hat{f x}\left(M_{0}, \hat{a}_{0}\right) \neq 0$, so that not all functions in $\mathfrak{F}$ vanish at a fixed point in $\mathfrak{M}(X) \times \hat{G}$.

(iv) Each function in $\mathfrak{F}$ vanishes at infinity in $\mathfrak{M}(X) \times \hat{G}$. For, suppose $\varepsilon>0$ is given. If $\sum_{i=1}^{n} \widehat{f_{i} x_{i}}(M, \hat{a}) \in \mathfrak{F}$, then

$$
\left|\sum_{i=1}^{n} \widehat{f_{i} x_{i}}(M, \hat{a})\right|=\left|\sum_{i=1}^{n} \hat{f}_{i}(\hat{a}) \phi_{M}\left(x_{i}\right)\right| \leqq \varepsilon
$$

if

$$
(M, \hat{a}) \notin\left(\bigcup_{i=1}^{n} \mathfrak{E}_{i}\right) \times\left(\bigcup_{i=1}^{n} \hat{C}_{i}\right) \equiv \Gamma
$$

where $\left|\hat{f}_{i}(\hat{a})\right|<\delta, \quad\left|\phi_{M}\left(x_{i}\right)\right|<\delta$ if $\hat{a} \notin \hat{C}_{i} \subset G$ and $M \notin \aleph_{i} \subset \mathfrak{M}(X)$. Here, $\delta<\min \left(\sqrt{\varepsilon / n}, \varepsilon / n K_{1}, \varepsilon / n K_{2}\right)$ with $K_{1}=\sup _{1 \leqq i \leqq n}\left|x_{i}\right|$ and $K_{2}=\sup _{1 \leqq i \leqq n} \sup _{\hat{a} \in \hat{\theta}}\left|\hat{f}_{i}(\hat{a})\right| ;$ $\hat{C}_{i}$ and $\mathfrak{C}_{i}$ are compact sets which exist because each $\hat{f}_{i}$ and each $x_{i}$ vanish at $\infty$ in $\hat{G}$ and $\mathfrak{M}(X)$, respectively. $\quad \Gamma$ is compact in $\mathfrak{M}(X) \times \hat{G}$ so that each function in $\mathfrak{F}$ vanishes at $\infty$.

We now appeal to a result in general point-set topology (see [5] $\mathrm{p}$. 12) which states: If $B$ is a family of complex-valued continuous functions vanishing at infinity on a locally compact space $S$, separating the points of $S$ and not all vanishing at any point of $S$, then the weak topology induced on $S$ by $B S$ is identical with the given topology of $S$. We take $S=\mathfrak{M}_{\mathfrak{C}}(X) \times \hat{G}$ and $\mathscr{G}=\mathfrak{F}$. This finishes the proof.

The next lemma deals with the radical and regularity in $B(G, X)$. Following this we conclude with the tauberian theorem.

Lemma 3. (i) The radical of $B$ consists of those functions $f \in B$ with values in the radical of $X$ a.e.

(ii) If $X$ is regular, then $B(G, X)$ is regular.

Proof. Necessity (i). Suppose $f$ takes values in the radical $\mathfrak{R}=\bigcap_{M \in \mathfrak{M}(X)} M$ of $X$ a.e. Then $\phi_{M} f=0$ a.e. for each $M \in \mathfrak{M}(X)$ and thus $\hat{f}(M, \hat{a})=0$ for each $(M, \hat{a}) \in \mathfrak{M}(X) \times \hat{G}$. This means $f$ is in the radical of $B$.

Sufficiency (i). Suppose that $f$ is in the radical of $B$. We must show that $f$ takes values in the radical $\Re$ of $X$, a.e. We have $\hat{f}(M, \hat{a})=0$ for all $(M, \hat{a}) \in \mathfrak{M}(X) \times \hat{G}$, that is $\int \phi_{M} f(a)(a, \hat{a}) d a=0$ for all $(M, \hat{a})$. Since $\phi_{M} f$ is in $L(G)$ and since $L(G)$ is semi-simple, we have $\phi_{M} f=0$ a.e. for each $M \in \mathfrak{M}(X)$. 
Let $\left\{j_{W}\right\}$ be an approximate identity for $L(G)$ consisting of bounded functions vanishing outside neighborhoods $W$ of the identity in $G$. Since $f$ is continuous in $B$-norm, it follows that the functions $j_{W} * f$ from $G$ to $X$ are continuous. Consequently, the functions $j_{W} * f$ take values in $\Re$ everywhere over $G$ since $\Re$ is closed in $X$. Choose a sequence $\left\{j_{W_{n}}\right\}$ from $\left\{j_{W}\right\}$ such that $j_{W_{n}} * f \rightarrow f$ in $B$-norm. Then, as is known, there is a subsequence of the $j_{W_{n}} * f$ converging to $f$ pointwise a.e. in $X$ norm. Since $\Re$ is closed, $f$ takes values in $\Re$ a.e.

Proof of (ii). Suppose $X$ is a regular algebra. We wish to show that, given any point $\left(M_{0}, \hat{a}_{0}\right) \in \mathfrak{M}(X) \times \hat{G}$ and any open set $\mathfrak{Q}$ containing $\left(M_{0}, \hat{a}_{0}\right)$, there is a function $g \in B(G, X)$ such that $\hat{g}\left(M_{0}, \hat{a}_{0}\right)=1$ and $\hat{g}(M, \hat{a})=0$ if $(M, \hat{a}) \notin \mathfrak{\Omega}$. By Lemma 2 , the open sets of $\mathfrak{M}(B)$ are of the form $\bigcup_{i \in \Omega}\left(\hat{O}_{i} \times \mathfrak{N}_{i}\right)$ where the $\hat{O}_{i}$ are open in $\hat{G}$ and the $\mathfrak{N}_{i}$ are open in $\mathfrak{M}(X)$. Suppose our $\mathfrak{Q}$ equals $\bigcup_{i \in \Omega}\left(\hat{O}_{i} \times \mathfrak{N}_{i}\right)$; then $\left(M_{0}, \hat{a}_{0}\right) \in \hat{O}_{i_{0}} \times \mathfrak{N}_{i_{0}}$ for some $i_{0} \in \Omega$, that is, $a_{0} \in \hat{O}_{i_{0}}$ and $M_{0} \in \mathfrak{R}_{i_{0}}$. We can find a function $f \in L(G)$ such that $\hat{f}\left(\hat{\alpha}_{0}\right)=1$ and $\hat{f}(\hat{a})=0$ if $\hat{a} \notin \hat{O}_{i_{0}}$. This follows from the regularity of the group algebra $L(G)$. Since $X$ is regular, by hypothesis, there is an $x \in X$ such that $\phi_{M_{0}}(x)=1$ and $\phi_{M}(x)=0$ if $M \notin \mathfrak{N}_{i_{0}}$. We will show that the $g$, above, can be taken to be $f x$. Firstly, $\hat{f x}\left(M_{0}, \hat{a}_{0}\right)=1$. Now, suppose $(M, \hat{a}) \notin \Omega$. Then $(M, \hat{a}) \notin \hat{O}_{i_{0}} \times \mathfrak{N}_{i_{0}}$ so that $\hat{a} \notin \hat{O}_{i_{0}}$ or $M \notin \mathfrak{N}_{i_{0}}$. In either case, $\hat{f x}(M, \hat{a})=0$. Hence $\hat{f} x(M, \hat{a})=0$ for all $(M, \hat{a}) \notin \precsim$.

We might add that if $B(G, X)$ is regular, then $X$ is likewise regular. However, this fact will not be used in the following theorem and so we do not enter into its proof.

Corollary. $B(G, X)$ is semi-simple if and only if $X$ is semi-simple.

Theorem. Let $X$ be semi-simple and regular. Suppose that the elements $x \in X$ with $\phi_{M}(x)$ having compact support in $\mathfrak{M}(X)$ are dense in $X$. Then every proper closed ideal in $B(G, X)$ is contained in a regular maximal ideal.

Proof. By the hypothesis and Lemma 3, it follows that $B(G, X)$ is regular and semi-simple. Using the general tauberian theorem (see the introduction), we can prove that any proper closed ideal in $B$ is contained in a regular maximal ideal by showing that if $f$ is any function in $B$ and $\varepsilon>0$, there exists an $h \in B$ such that $\|f-h\|_{B} \leqq \varepsilon$ and $\hat{h}(M, \hat{a})$ has compact support in $\mathfrak{M}(X) \times \hat{G}$. Suppose, therefore, that $f \in B$ and $\varepsilon>0$ are given. We can find $f_{i} \in L(G), x_{i} \in X(i=1,2, \cdots, n)$, such that 


$$
\left\|f-\sum_{i=1}^{n} f_{i} x_{i}\right\|_{B}<\varepsilon / 3
$$

We have functions $f_{i}^{\prime} \in L(G)$ such that $\left|f_{i}-f_{i}^{\prime}\right|_{L}<\varepsilon / 3 K n(i=1,2, \cdots, n)$, where $K=\sup _{1 \leq i \leqq n}\left|x_{i}\right|$ and the $\hat{f}_{i}^{\prime}$ have compact support $\hat{C}_{i} \subset \hat{G}$. This follows from the fact that $L(G)$ satisfies the hypotheses of the general tauberian theorem. By the hypotheses on $X$, we may find $x_{i}^{\prime}$ in $X$ such that $\left|x_{i}-x_{i}^{\prime}\right|<\varepsilon / 3 R n(i=1,2, \cdots, n)$, where $R=\sup _{1 \leqq i \leqq n}\left|f_{i}^{\prime}\right|_{L}$ and the $\phi_{M}\left(x_{i}\right)$ have compact support $\mathbb{C}_{i} \subset \mathfrak{M}(X)$. Now

$$
\begin{array}{r}
\left\|f-\sum_{i=1}^{n} f_{i}^{\prime} x_{i}^{\prime}\right\|_{B}=\left\|f-\sum_{i=1}^{n} f_{i} x_{i}+\sum_{i=1}^{n}\left(f_{i}-f_{i}^{\prime}\right) x_{i}+\sum_{i=1}^{n} f_{i}^{\prime}\left(x_{i}-x_{i}^{\prime}\right)\right\|_{B} \\
\leqq \varepsilon / 3+K n(\varepsilon / 3 K n)+R n(\varepsilon / 3 R n)=\varepsilon .
\end{array}
$$

Take (see above) $h \equiv \sum_{i=1}^{n} f_{i}^{\prime} x_{i}^{\prime}$. We see that $\hat{h}(M, \hat{a})$ has support $\left(\bigcup_{i=1}^{n} \mathfrak{S}_{i}\right) \times\left(\bigcup_{i=1}^{n} \hat{C}_{i}\right)$ which is compact in $\mathfrak{M}(X) \times \hat{G}$. The theorem is now proved.

\section{REFERENCES}

1. S. Bochner, Integration von Funktionen, deren Werte die Elemente eines Vektorraumes sind. Fund. Math., 20 (1933), 262-276.

2. A. Hausner, Ideals in a certain Banach algebra, Proc. Amer. Math. Soc., 8 (1956), 246-249.

3. _- Group algebras of vector-valued functions, Bull. Amer. Math. Soc., 62 (1956), abstract 493 .

4. E. Hille, Functional analysis and semi-groups, New York, 1948.

5. L. H. Loomis, An introduction to abstract harmonic analysis, New York, 1953.

6. G. W. Mackey, Commutative Banach algebras, (edited by A. Blair), mimeographed notes, Harvard University, 1952.

The City College of New York 


\title{
PACIFIC JOURNAL OF MATHEMATICS
}

\section{EDITORS}

H. L. RoYden

Stanford University

Stanford, California

\section{R. A. Beaumont}

University of Washington

Seattle 5 , Washington
A. L. Whiteman

University of Southern California

Los Angeles 7, California

E. G. Straus

University of California

Los Angeles 24, California

\section{ASSOCIATE EDITORS}

\author{
E. F. BECKENBACH \\ C. E. BURGESS \\ M. HALL \\ E. HEWITT
}
A. HORN
V. GANAPATHY IYER
R. D. JAMES
M. S. KNEBELMAN

L. NACHBIN

I. NIVEN

T. G. OSTROM

M. M. SCHIFFER
G. SZEKERES

F. WOLF

K. YOSIDA

\section{SUPPORTING INSTITUTIONS}

\author{
UNIVERSITY OF BRITISH COLUMBIA \\ CALIFORNIA INSTITUTE OF TECHNOLOGY \\ UNIVERSITY OF CALIFORNIA \\ MONTANA STATE UNIVERSITY \\ UNIVERSITY OF NEVADA \\ OREGON STATE COLLEGE \\ UNIVERSITY OF OREGON \\ UNIVERSITY OF SOUTHERN CALIFORNIA
}

Mathematical papers intended for publication in the Pacific Journal of Mathematics should be typewritten (double spaced), and the author should keep a complete copy. Manuscripts may be sent to any of the editors. All other communications to the editors should be addressed to the managing editor, E. G. Straus at the University of California, Los Angeles 24, California.

50 reprints per author of each article are furnished free of charge; additional copies may be obtained at cost in multiples of 50 .

The Pacific Journal of Mathematics is published quarterly, in March, June, September, and December. The price per volume (4 numbers) is $\$ 12.00$; single issues, $\$ 3.50$. Back numbers are available. Special price to individual faculty members of supporting institutions and to individual members of the American Mathematical Society: $\$ 4.00$ per volume; single issues, $\$ 1.25$.

Subscriptions, orders for back numbers, and changes of address should be sent to Pacific Journal of Mathematics, 2120 Oxford Street, Berkeley 4, California.

Printed at Kokusai Bunken Insatsusha (International Academic Printing Co., Ltd.), No. 10, 1-chome, Fujimi-cho, Chiyoda-ku, Tokyo, Japan.

PUBLISHED BY PACIFIC JOURNAL OF MATHEMATICS, A NON-PROFIT CORPORATION

The Supporting Institutions listed above contribute to the cost of publication of this Journal, but they are not owners or publishers and have no responsibility for its content or policies. 


\section{Pacific Journal of Mathematics}

\section{Vol. 7, No. 4 \\ April, 1957}

Robert Geroge Buschman, A substitution theorem for the Laplace

transformation and its generalization to transformations with

symmetric kernel .

S. D. Conte, Numerical solution of vibration problems in two space variables ...................................... 1535

Paul Dedecker, A property of differential forms in the calculus of variations ......................................... 1545

H. Delange and Heini Halberstam, A note on additive functions . . . . . . . 1551

Jerald L. Ericksen, Characteristic direction for equations of motion of non-Newtonian fluids ................................. 1557

Avner Friedman, On two theorems of Phragmén-Lindelöf for linear elliptic and parabolic differential equations of the second order ........... 1563

Ronald Kay Getoor, Additive functionals of a Markov process . . . . . . . . . 1577

U. C. Guha, $(\gamma, k)$-summability of series . . . . . . . . . . . . . . . . . . 1593

Alvin Hausner, The tauberian theorem for group algebras of vector-valued functions ...................................... 1603

Lester J. Heider, $T$-sets and abstract $(\mathrm{L})$-spaces ................. 1611

Melvin Henriksen, Some remarks on a paper of Aronszajn and

Panitchpakdi........................................ 1619

H. M. Lieberstein, On the generalized radiation problem of A. Weinstein ... 1623

Robert Osserman, On the inequality $\Delta u \geq f(u) \ldots \ldots \ldots \ldots \ldots \ldots \ldots . \ldots 1641$

Calvin R. Putnam, On semi-normal operators . . . . . . . . . . . . . . . . . . 1649

Binyamin Schwarz, Bounds for the principal frequency of the

non-homogeneous membrane and for the generalized Dirichlet integral...

Edward Silverman, Morrey's representation theorem for surfaces in metric

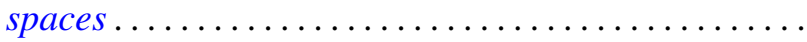

V. N. Singh, Certain generalized hypergeometric identities of the Rogers-Ramanujan type. II .

R. J. Smith, A determinant in continuous rings 1701

Drury William Wall, Sub-quasigroups of finite quasigroup 1711

Sadayuki Yamamuro, Monotone completeness of normed semi-ordered linear spaces.

C. T. Rajagopal, Simplified proofs of "Some Tauberian theorems" of Jakimovski: Addendum and corrigendum ............

N. Aronszajn and Prom Panitchpakdi, Correction to: "Extension of uniformly continuous transformations in hyperconvex metric spaces"...................................

Alfred Huber, Correction to: "The reflection principle for polyharmonic functions" 